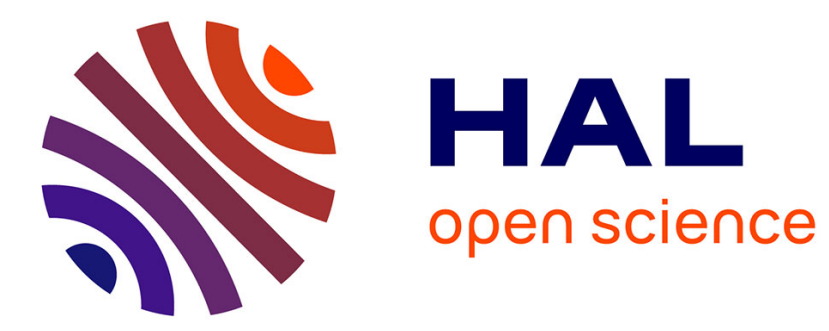

\title{
Formes et sens de la relation concessive dans le Débat de Folie et d'Amour de Louise Labé (1555)
}

Véronique Montagne

\section{To cite this version:}

Véronique Montagne. Formes et sens de la relation concessive dans le Débat de Folie et d'Amour de Louise Labé (1555). L'information grammaticale, 2004, 104, pp.37-41. hal-01363058

\section{HAL Id: hal-01363058 \\ https://hal.univ-cotedazur.fr/hal-01363058}

Submitted on 23 Jan 2020

HAL is a multi-disciplinary open access archive for the deposit and dissemination of scientific research documents, whether they are published or not. The documents may come from teaching and research institutions in France or abroad, or from public or private research centers.
L'archive ouverte pluridisciplinaire HAL, est destinée au dépôt et à la diffusion de documents scientifiques de niveau recherche, publiés ou non, émanant des établissements d'enseignement et de recherche français ou étrangers, des laboratoires publics ou privés. 


\section{Formes et sens de la relation concessive dans le Débat de Folie et d'Amour de Louise Labé (1555)}

$\mathrm{Au}$ XVIème siècle, la réflexion grammaticale sur la concession reste essentiellement descriptive. Les auteurs s'intéressent surtout aux outils logiques utilisés dans ce type de relation logique. Dans son Tretté de la grammere francoeze (1550), Louis Meigret répertorie ainsi certains connecteurs concessifs sous la rubrique des conjonctions adversatives :

Les aocunes sont auersatives : come, més, toutefoes, combien que, a tout le moins, ains, ainçoes $^{1}$

Dans L'éclaircissement de la langue française $(1530)^{2}$, Palsgrave, cite pareillement un certain nombre de marqueurs concessifs, qui fonctionnent en corrélation, comme combien quel toutefois, combien quel si, ou jasoyt ce quel neantmoins.

Ces considérations grammaticales n'impliquent qu'une analyse sommaire de la relation logique, sans commune mesure avec la réflexion ramiste ${ }^{3}$. Dans sa Grammaire (1562, puis 1572), Pierre de la Ramée classe les marqueurs concessifs dans la catégorie générale des conjonctions énonciatives, dans laquelle «les parties sont asseurées pour certain». A l'intérieur de la catégorie énonciative, il isole le groupe des conjonctions ségrégatives, "quant les sens comme non-vrays ensembles sont segregés». Enfin, dans l'ensemble des conjonctions ségrégatives, il relève la catégorie des conjonctions discrétives, "quand les parties sont separées de raison » et il illustre ce cas de figure par les marqueurs concessifs " combien-que », «toutesfois », «mais», «atoutlemoins », « «ains », " pour le moins », ou encore « iacois $»^{4}$.

Dans sa Dialectique (1555), il propose la même approche de la concession : la relation logique est classée dans les arguments artificiels, et plus spécifiquement dans la catégorie des comparez. Dans les comparez, il distingue deux ensembles: le premier concerne les comparaisons quantitatives, le second est d'ordre qualitatif. Dans ce dernier ensemble, il identifie l'argument des dissemblables, présenté comme suit :

Dissemblables sont desquelz la qualité est diverse et s'appellent aussi différentz, comme dissimilitude et différence sont prises pour un ${ }^{5}$

La plupart des exemples fournis comportent des marqueurs concessifs. Ramus explique ainsi que "la dissimilitude est fort fréquente en ceste formule : Combien...toutefois », ou encore avec la corrélation «si...néantmoins ».

Dans le Débat de Folie et d'Amour ${ }^{6}$ (1555), Louise Labé utilise la concession de façon assez conséquente. Dans un premier temps, nous décrirons les divers outils concessifs mis en oeuvre, en établissant notamment des comparaisons avec les pratiques alors les plus usuelles. Puis, nous analyserons deux effets de sens de cette relation logique, en nous référant notamment à l'approche ramiste.

\section{LES FORMES DE LA CONCESSION}

La concession peut prendre place dans une proposition dépendante, ou non-dépendante.

\footnotetext{
${ }^{1}$ Meigret, $1970: 177$.

2 Palsgrave, 1852 : 872 .

${ }^{3}$ Voir Chevalier, $1968: 284$.

${ }^{4}$ Ramus, $2001: 102-103$.

${ }^{5}$ Ramus, $1996: 87$.

${ }^{6}$ L'édition de référence est celle de François Rigolot, parue chez GF-Flammarion, en 1986.
} 


\subsection{Hypotaxe :}

Dans La concession dans la phrase complexe en français des origines au XVIème siècle, O.Soutet s'intéresse notamment aux conjonctions de subordination de sens concessif les plus utilisées au XVIème siècle. Pour les périodes 1500-1549 et 1550-1599, il propose les chiffres suivants ${ }^{7}$ :

\begin{tabular}{|l|l|l|}
\hline Locution & $\mathbf{1 5 0 0 - 1 5 4 9}$ & $\mathbf{1 5 5 0 - 1 5 9 9}$ \\
\hline BIEN QUE & $8,5 \%$ & $25 \%$ \\
\hline COMBIEN QUE & $59 \%$ & $35 \%$ \\
\hline JA SOIT (CE) QUE & $1,5 \%$ & $1,75 \%$ \\
\hline ENCORE QUE & $\mathrm{N}$ & $30 \%$ \\
\hline NEANMOINS QUE & $3,5 \%$ & $\mathrm{~N}$ \\
\hline (CE) NONOBSTANT QUE & $20,5 \%$ & $1,75 \%$ \\
\hline QUOI QUE & $7 \%$ & $6,5 \%$ \\
\hline
\end{tabular}

Dans le Débat, Louise Labé utilise essentiellement combien que et encore que, dans les proportions relatives suivantes :

\begin{tabular}{|l|l|l|}
\hline Locutions & Nombre d'occurrences & Rapport \\
\hline COMBIEN QUE & 9 & $34,7 \%$ \\
\hline ENCORE QUE & 17 & $65,3 \%$ \\
\hline
\end{tabular}

Si l'on tient compte des deux autres structures hypotaxiques présentes dans notre corpus ${ }^{8}$, les proportions sont les suivantes :

\begin{tabular}{|l|l|l|}
\hline Locutions & Nombre d'occurrences & Rapport \\
\hline COMBIEN QUE & 9 & $19,5 \%$ \\
\hline ENCORE QUE & 17 & $36,9 \%$ \\
\hline QUAND & 5 & $10,8 \%$ \\
\hline Relative indéfinie & 15 & $32,6 \%$ \\
\hline
\end{tabular}

On peut d'ores et déjà signaler deux traits originaux : une proportion inhabituelle, à l'époque, de la locution encore que et une utilisation importante des relatives indéfinies.

\subsubsection{Encore que ${ }^{10} /$ combien que}

Les deux locutions conjonctives apparaissent dans trois types de configuration syntaxique : elles introduisent une subordonnée qui peut être placée avant ou après la proposition principale, ou encore à l'intérieur de cette principale.

\section{Subordonnée + principale :}

Dans les cas où la proposition subordonnée est antéposée, la concession est de type logique, selon la typologie de M-A.Morel utilisée $\mathrm{ici}^{11}$. Cela signifie que la subordonnée, normalement associée à une principale $\mathrm{B} 1$, est en l'occurrence associée à une principale $\mathrm{B}$, qui contredit précisément B1. Ainsi, dans l'exemple «Et combien qu'il en pousse l'une, pinse l'autre,

\footnotetext{
${ }^{7}$ Soutet, 1992 : 211. La lettre $\mathrm{N}$ utilisée dans le tableau indique que la fréquence des occurrences n'est pas signalée par O.Soutet, parce que non significative dans son corpus.

${ }^{8}$ Voir infra.

${ }^{9}$ A titre de comparaison, signalons que, dans l'Heptaméron (1559) de Marguerite de Navarre, c'est la locution conjonctive combien que qui est la plus utilisée (83,3\%). Elle est suivie par encore que (11,53\%), puis par nonobstant que $(5,12 \%)$. Voir Montagne, 2003.

${ }^{10}$ La locution est orthographiée encore que ou encor que.

${ }^{11}$ Voir Morel, 1996.
} 
descoiffe, leve la cotte, et leur face mile maus : si le chercheront elles tousjours $»^{12}$ (p.91), le fait introduit dans la subordonnée, à savoir "il en pousse l'une, pinse l'autre, descoiffe, leve la cotte, et leur face mile maus » est normalement associé à «elles ne le chercheront pas/ pas toujours ». La principale «si le chercheront elles tousjours » contredit la conclusion attendue , ce qui a pour effet de renforcer l'assertion de B.

Dans ce type de configuration, et conformément à ce que remarque Palsgrave ${ }^{13}$, la principale est souvent introduite par une corrélation, en l'occurrence si, si est ce que ou toutefois.

\section{*En corrélation :}

La corrélation si est suivie d'une inversion du sujet et du verbe :

Car encore que je puisse savoir de moysmesme la verité du fait, si ne veus je point mettre en avant cette coutume qui pourroit tourner à consequence (p.60) ${ }^{14}$

Car combien qu'il soit des grans amis de Venus, si suis je seure, que s'il entreprent parler pour moy, il n'oublira rien qui serve à ma cause $(\mathrm{p} .60)^{15}$

Avec si est ce que, qui comporte en lui-même une inversion sujet-verbe, et toutefois, l'ordre sujet-verbe est rétabli dans la principale. Le terme corrélatif est placé en tête de principale dans le cas de si est-ce que :

Encore que vous m'ayez tant montré de faveur et d'amitié, si est ce que je n'ose vous suplier... (p.59)

Et combien que nature ne lui ust nié plusieurs graces [...], si est ce qu'elle ne vouloit plaire à autre qu'à celui qui la tenoit prisonniere. $(\mathrm{p} .97)^{16}$

Il est placé après le groupe sujet-verbe dans le cas de toutefois :

Car combien que Folie ait premierement parlé à Amour, ce n'estoit toutefois pour quereler (p.83)

\section{*Hors corrélation :}

On note deux cas de non-corrélation, où la subordonnée concessive antéposée est juxtaposée avec une subordonnée hypothétique introduite par si, la conjonction de subordination empêchant l'utilisation d'une éventuelle corrélation en si en début de principale :

Si tu usses esté plus modeste, encore que je te fusse inconnue : cette faute ne te fust avenue (p.54)

Et combien qu'elles vousissent prier, si elles osoient, elles se laissent adorer (p.96)

\section{Principale + subordonnée :}

Dans les occurrences suivantes, la concession peut relever du type logique, dont l'ordre de succession des propositions est relativement libre ${ }^{17}$. Contrairement à ce qui est le plus usuel en français contemporain, la locution conjonctive encore que semble ici avoir régulièrement cette valeur logique, plutôt que rectificative ${ }^{18}$ :

Par pitié on baille à manger à une creature, encore qu'elle n'en demande (p.73) ${ }^{19}$

Ce n'est pas par ta force et vertu, que tant de miracles soient avenuz au monde : mais par mon industrie, par mon moyen et intelligence : combien que tu ne me connoisses (p.51)

\footnotetext{
${ }^{12}$ Souligné par nous, comme dans les exemples qui suivent.

${ }^{13}$ Voir supra et Lorian, $1973: 187$ sq.

${ }^{14}$ Voir aussi p.61, 82.

${ }^{15}$ Voir aussi p.91 (x2).

${ }^{16}$ Voir aussi l'épître dédicatoire, p.42.

${ }^{17}$ Voir Morel, 1996 : 8.

${ }^{18}$ Voir infra.

${ }^{19}$ Voir aussi p.67, 73 (x2), 76, 87, 89, 95 (x2), 102.
} 
La concession peut aussi avoir un sens rectificatif ${ }^{20}$. La principale, nécessairement antéposée et normalement associée à une subordonnée A1, est alors utilisée avec une subordonnée qui contredit A1. Ainsi, dans l'exemple "Pour le moins te devroient ils haïr, encore qu'ils ne t'osassent offenser» (p.62), la principale "Pour le moins te devroient ils haïr " est normalement associée à la proposition «ils osent t'offenser». Or la subordonnée concessive rectifie cette conclusion attendue et la portée de la principale s'en trouve limitée. La rectification peut porter sur l'utilisation d'un terme, en l'occurrence de l'adjectif ancienne :

Demeure donq en paix, Amour : et ne vien rompre l'ancienne ligue qui est entre toy et moy : combien que tu n'en susses rien jusqu'à present (p.102)

\section{Subordonnée insérée dans la principale :}

Dans ce dernier cas de figure, la concession, en l'occurrence logique, peut être insérée entre le support agentif d'une proposition infinitive, et le verbe à l'infinitif :

Vous verrez les Sages mesmes, encore qu'il soit dit que lon cherche son semblable, tomber de ce coté (p.91)

Ou bien entre un verbe, en l'occurrence la forme voulant, et son deuxième COD qu'ils lui fussent neanmoins inutiles ${ }^{21}$ :

Mais la traytresse lui mit un tel bandeau, que jamais ne sera possible lui oter : par ce moyen voulant se moquer de toute l'ayde que tu lui pourrois donner : et encor que tu lui rendisses les yeus, qu'ils lui fussent neanmoins inutiles (p.66)

\subsubsection{Quand}

Il faut également signaler quelques occurrences de la conjonction quand suivie du conditionnel ou du subjonctif, utilisé dans les deux parties de la concession. Dans les deux cas, la proposition cumule les effets de l'hypothèse et de la concession et quand est l'équivalent de même si :

De si foible taille que quand j'aurois un bras lié, si ne te creindrois je gueres (p.49)

Quand elle lui ust pis fait, je ne voy point comment on lui en ust pù rien demander $(\mathrm{p} .83)^{23}$

\subsubsection{Les tours complexes}

La concession peut enfin être exprimée dans une subordonnée relative indéfinie. Avec la corrélation quelque...que, la protase concessive permet une focalisation, qu'elle porte sur le degré d'intensité de la qualité (quelque + adjectif : concession scalaire) ou sur l'identité d'un élément à l'intérieur d'une classe (quelque + substantif : concession extensionnelle) ${ }^{24}$. Dans les deux cas, la concession est de type logique ${ }^{25}$.

La concessive extensionnelle en quelque + substantif + que induit "l'idée d'un "tirage aléatoire " effectué au sein d'une classe référentielle (on peut sélectionner n'importe lequel des éléments de la classe), le choix étant seulement contraint par le caractère prototypique de l'élément sélectionnable ${ }^{26}$. Quelque a le statut d'un déterminant du nom, il peut donc être au singulier ou au pluriel :

\footnotetext{
${ }^{20}$ Voir Morel, $1996: 10 s q$.

${ }^{21}$ Voulant est construit avec un double complément : le groupe infinitival «se moquer de toute l'ayde que tu lui pourrois donner » et la complétive "qu'ils lui fussent neanmoins inutiles ». Encore que a ici le sens de «même si ».

${ }^{22}$ Voir aussi p.52-53, 101.

${ }^{23}$ Voir aussi p.84.

${ }^{24}$ Voir Morel : 115 .

${ }^{25}$ Ibid.

${ }^{26}$ Ibid : 124.
} 
Qu'ainsi soit dit, que tous ceus qui aymeront (quelque faveur qu'ils ayent) ne soient sans mal, et infortune (p.58) ${ }^{27}$

$\mathrm{Ne}$ les áy je fait sortir de leurs abimes, et venir espouventer les humains, et ravir les filles à leurs meres, quelques juges qu'ils soient de telz forfaits et transgressions faites contre les loix ? (p.50)

On note deux cas de corrélation, avec toutefois ou si :

Et ceus qui ont esté excellens Poëtes, ou en ont tout rempli leurs livres, ou, quelque autre suget qu'ils ayent pris, n'ont osé toutefois achever leur euvre sans en faire honorable mencion (p.77)

quelque grand degré ou je sois, si ay je esté bien peu aymé (p.63)

Du point de vue sémantique, la relative indéfinie introduite par quoi ou par quiconque, fonctionne comme quelque + substantif + que. La concession, qui affecte l'identité d'un élément à l'intérieur d'une classe, est introduite par le pronom indéfini non animé quoi, suivi du pronom relatif que, ou du «pronom relatif » animé quiconque :

Estant Amour desir, ou, quoy que ce soit, ne pouvant estre sans desir : il faut confesser qu'incontinent que cette passion vient saisir l'homme, elle l'altere et immue (p.99) ${ }^{29}$

Aumoin di moy, quinconque sois, si à l'aventure ma flesche t'a frapee, et si elle t'a blessee (p.52)

D'un point de vue diachronique, on remarque que la concession est doublement exprimée dans ce dernier exemple : d'abord avec aumoin, qui se rapproche du groupe à tout le moins classé dans les marqueurs concessifs par Meigret et Ramus ${ }^{30}$, ensuite par la relative indéfinie.

La concession scalaire en quelque + adjectif + que porte sur le degré d'intensité de la qualité évoquée. Le locuteur effectue un parcours sur une échelle d'intensité, en direction du degré maximal $^{31}$. Dans la proposition relative, l'attribut est antéposé au sujet ou au COD auquel il se rapporte :

Quelque petit que tu me voyes, suis le plus creint et redouté entre les Dieus et les hommes $(\mathrm{p} .50)^{32}$

\subsection{Parataxe :}

\subsubsection{Toutefois / néanmoins}

O.Soutet note, qu'au XVIème siècle, les adverbes les plus utilisés sont toutefois (75\%), néantmoins (18\%), suivis de loin par tote $(s)$ voie $(s)$ et nonportant $(7 \%)$. Louise Labé utilise surtout néanmoins (43,3\% des cas), puis toutefois (26,6\%), si et si est ce que (20\%) et enfin encore (10\%). Là aussi, un outil concessif prévaut sur les autres, en l'occurrence néanmoins, plus utilisé par Louise Labé que par ses contemporains.

Selon M-A.Morel, les adverbes toutefois et néanmoins introduisent des concessions logique ou rectificative: "si c'est la première proposition qui est interprétable comme une subordonnée concessive, [ces adverbes] marquent une relation à valeur de concession logique ${ }^{33}$. C'est le cas dans les exemples suivants :

\footnotetext{
${ }^{27}$ Voir aussi p.69, 74, 96.

${ }^{28}$ Voir aussi l'épître dédicatoire, p.42 (x2).

${ }^{29}$ Le début de l'exemple est à comprendre de la façon suivante : «l'amour est un désir, ou quelque chose d'autre, mais ce quelque chose est toujours accompagné de désir ».

${ }^{30}$ Voir supra. La remarque vaut également pour l'exemple «Pour le moins te devroient ils hä̈, encore qu'ils ne t'osassent offenser» (p.62).

${ }^{31}$ Voir Morel, $1996: 116$.

${ }^{32}$ Voir aussi p.51, 70, 76, 78, 89 .

${ }^{33}$ Morel, 1996 : 54.
} 
si cette outrecuidee ha fait quelque desordre si pres de ma personne, il lui sera cher vendu. Toutefois il la faut ouir... (p.59-60) [= Bien que cette outrecuidée ait fait quelque désordre si près de ma personne, il la faut ouï $\left.{ }^{34}\right]^{35}$

Lui semble [...] que serez tous si fols, que de l'absoudre. Je n'ay neanmoins charge par Amour de requerir vengeance et punition de Folie (p.67) ${ }^{36}$

Il est à noter que la relation concessive peut s'instaurer, non pas au niveau purement référentiel des deux propositions coordonnées, mais au niveau des conclusions que le locuteur peut tirer de l'une des propositions ${ }^{37}$. En voici un exemple :

Folie ha premierement mis en teste à quelcun de se faire creindre : Folie ha fait les autres obeïr [...] Et neanmoins, qui ha il plus venerable entre les hommes, que ceus qui commandent aus autres ? (p.86)

Il faut supposer ici la conclusion implicite, tirée par l'interlocuteur au terme de la première proposition : « la Folie est une mauvaise chose, donc ce qu'elle commande est répréhensible ». La concession en néanmoins vient contredire cette conclusion.

Dans le cas où ces adverbes introduisent une nuance à la première proposition énoncée, la concession est rectificative :

me voici preste à respondre à tout ce qu'Amour me voudra demander. Toutefois j'ay une requeste à te faire (p.60) [Me voici prête à répondre à tout ce qu'Amour voudra me demander, encore que $\mathrm{j}$ 'aie une requête à te faire $]^{38}$

Il ne lui ha fait mal : neanmoins il s'en est mis en son plein devoir ${ }^{39}(\mathrm{p} .83)^{40}$

Le partage, souvent délicat, entre concession logique et rectificative, est parfois rendu possible par la connaissance du contexte et du locuteur concerné. Ainsi, l'exemple "Il ne lui ha fait mal : neanmoins il s'en est mis en son plein devoir » sera plutôt interprété comme une concession rectificative, dans la mesure où il s'agit d'un argument de Mercure contre Amour $^{41}$. La concession rectifie une possible conclusion en faveur de son adversaire.

\subsubsection{Si / si est ce que}

Dans la Grammaire de l'ancien français, G.Moignet précise que si peut avoir une valeur adversative assez forte qui en fait l'équivalent de pourtant ${ }^{42}$. C.Marchello-Nizia fait observer que cette valeur adversative est celle qui persistera le plus longtemps, quand seront oubliées, par exemple, les valeurs causales ou consécutives ${ }^{43}$. Elle définit le terme de la façon suivante : "Si est le marqueur de la coexistence, dans l'univers du locuteur, de deux vérités qui, pour un interlocuteur potentiel, paraîtraient exclusives l'une de l'autre ${ }^{44}$.

En l'occurrence, la concession est plutôt rectificative ${ }^{45}$ :

\footnotetext{
${ }^{34}$ Dans cet exemple, la condition "si cette outrecuidee ha fait quelque desordre si pres de ma personne » est tenue pour un acquis au début de la proposition concessive : "cette outrecuidée ha fait quelque desordre », elle sera punie ( il lui sera cher vendu »), mais il faut quand même l'écouter.

${ }^{35}$ Voir aussi p.62.

${ }^{36}$ Voir aussi p.60, 65, 68, $86(x 2), 98$.

${ }^{37}$ Voir Morel, $1996: 86$.

${ }^{38}$ Voir aussi p.60-61, 82, 89 (x2), 97.

39 «Il s'en est mis en son plein devoir» est à comprendre comme « il a fait tout ce qu'il fallait pour lui faire mal ».

${ }^{40}$ Voir aussi p.61, 72, 86, 87, 94.

${ }^{41}$ Voir infra.

${ }^{42}$ Moignet, 1988 : 288.

${ }^{43}$ Marchello-Nizia, 1985 : 356.

${ }^{44}$ Ibid., p. 140.

${ }^{45}$ Voir aussi l'épître dédicatoire, p.42.
} 
J'ay bien dit que je fay aymer encor ceus, qui ne sont point aymez : mais si est il en la puissance d'un chacun le plus souvent de se faire aymer (p.63)

\subsubsection{Encore}

Cette particule, de valeur temporelle à l'origine, sert assez tôt à exprimer la valeur concessive, en l'occurrence rectificative. Comme en français moderne, elle apparaît alors plutôt en tête de phrase ou de proposition, éventuellement derrière « et », et toujours avec une inversion sujetverbe :

Les montaignes de Sicile couvrent elles de plus execrables personnes ? Et encores n'a elle honte de se presenter devant voz divinitez (p.67)

Aussi tot un autre aura faveur de parler à elle que lui, qui ha esté convié. Encore ha il occasion de se contenter, à son avis (p.96) ${ }^{47}$

Signalons enfin que l'adverbe pourtant doit ici être exclu de ce relevé :

Reste de te prier, Jupiter, et vous autres Dieus, de n'avoir point respect aus noms [...] mais regarder à la verité et dinité des choses. Et pourtant, s'il est plus honorable entre les hommes dire un tel ayme, que, il est fol: que celà leur soit imputé à ignorance (p.102)

Comme le fait observer O.Soutet ${ }^{48}$, la spécialisation concessive de pourtant n'est pas acquise au XVIème siècle, et François Rigolot signale ainsi, dans son glossaire, que pour tant est ainsi l'équivalent de pour cela ou, en l'occurrence de pour cette raison, par conséquent ${ }^{49}$.

\subsection{Tours argumentatifs}

La concession argumentative ${ }^{50}$ est formée de deux propositions coordonnées. La première comporte, en l'occurrence, un constituant verbal (dire) ou adjectival (vrai) qui témoigne de l'accord de principe du locuteur sur une opinion, une assertion, par la suite nuancée par la seconde proposition introduite par mais :

j'ay bien dit que je fay aymer encore ceus, qui ne soint point aymez: mais si est il en la puissance d'un chacun le plus souvent de se faire aymer (p.63)

Vray est qu'au commencement les hommes ne faisoient point de hautes folies, aussi n'avoient ils encores aucuns exemples devant eus. Mais leur folie estoit à courir l'un apres l'autre (p.85)

\section{LES DEBOUCHES STYLISTIQUES}

En guise de conclusion, nous observerons ici deux effets de sens de la relation concessive. En effet, la concession peut être utilisée à diverses fins : ce peut être une forme de l'amplification, ce peut aussi être un argument réfutatif.

\subsection{Concession \& dissimilitude}

La dissimilitude évoquée par Ramus est mise en évidence par la présence d'antonymes qui montrent que des « sens comme non-vrays ensembles sont segregés » ${ }^{52}$ :

\footnotetext{
${ }^{46}$ Voir aussi p.62, 99, 86, 93. Pour cet exemple de la p.63, voir aussi infra.

${ }^{47}$ Voir aussi p.96.

${ }^{48}$ Soutet, $1992: 224$.

${ }^{49}$ On peut gloser l'exemple de la façon suivante : «Ne vous fiez pas au prestige attaché au nom d'Amour ou au mépris attaché au nom de Folie. Et, par conséquent, si les hommes considèrent comme plus honorable de dire de quelqu'un «il est amoureux », que de dire «il est fou », cela relève de l'ignorance (de la méconnaissance des qualités de Folie).

${ }^{50}$ Voir Morel, 1996 : 15 sq.

${ }^{51}$ Pour l'analyse de cet exemple, voir supra.
} 
Pour le moins te devroient ils hä̈r [...]. Toutefois tous tant qu'ils sont t'ayment. (p.62)

Ou par l'opposition entre une proposition négative et une proposition affirmative :

A cette cause, choisi quel autre tu voudras pour parler pour vous : et croy qu'il ne lui sera besoin lui commander [...]. Neanmoins s'il en est besoin, je lui commanderay (p.61)

Les notions rapprochées dans la concession sont tenues pour contradictoires, et il arrive que les locuteurs justifient, d'une façon ou d'une autre, la coexistence logique des deux thèses proposées, pour lever le paradoxe que leur présence conjointe implique. Dans le texte de Louise Labé, l'incompatibilité peut être justifiée par une notion de cause :

C'est chose bien dure à Mercure moyenner desplaisir à Venus. Toutefois, puis que tu me contreins, je feray mon devoir... (p.60-61) ${ }^{53}$

Ou une hypothèse :

Car combien qu'il soit des grans amis de Venus, si suis je seure, que s'il entreprent parler pour moy, il n'oublira rien qui serve à ma cause (p.60)

La justification des dissimilitudes est un moyen de préserver la cohérence des propos tenus en restreignant la validité de l'énoncé concédé. Quand un locuteur ne justifie par l'incompatibilité entre les deux faits mis en relation, c'est essentiellement à des fins d'amplification. C'est ainsi que Jupiter manifeste son étonnement face aux comportements des dieux et des hommes à l'encontre d'Amour :

Pour le moins te devroient ils hä̈r [...]. Toutefois tous tant qu'ils sont t'ayment. (p.62) ${ }^{55}$

\subsection{Concession \& réfutation}

Dans la relation concessive rectificative ou logique, l'association présupposée entre une cause et sa conséquence est invalidée. Ainsi, dans la proposition «On pense à un malade, encore qu'il ne veuille guerir » (p.73), l'énoncé concédé « il ne veut guérir » est normalement associé à la proposition « on ne le panse pas ». Or cette conclusion est en l'occurrence invalidée par la proposition «on pense à un malade » (= on panse un malade). Le présupposé qui associe les deux parties de l'énoncé est ici remis en question, tout en étant nécessairement admis par l'interlocuteur, sans quoi la concession n'aurait évidemment aucun sens.

Les phrases concessives permettent donc de décoder certains présupposés propres à un texte, à une époque, à une situation. Dans l'énoncé suivant, on doit ainsi présupposer que le fait de s'adresser à quelqu'un «premierement » (« en premier»), en est normalement associé au fait de chercher querelle :

Car combien que Folie ait premierement parlé à Amour, ce n'estoit toutefois pour quereler (p.83)

La concession permet de manifester, au moins temporairement, un accord de principe avec des opinions partagées par le locuteur. Cette marque de respect peut expliquer, en partie, l'importance de la concession dans le discours de Mercure qui, à lui seul, utilise $48 \%$ des exemples concessifs. Dans la mesure où, de son propre aveu, il défend une mauvaise cause ${ }^{56}$, un type de cause que Fabri qualifie de "deshonneste » dans son Grand et vrai art de pleine rhétorique $(1521)^{57}$, il est utile qu'il prenne les précautions requises pour se concilier un

\footnotetext{
${ }^{52}$ Voir supra.

${ }^{53}$ Voir aussi p.73.

${ }^{54}$ Voir aussi p.61.

${ }^{55}$ Voir aussi p.73 (x2), 50, 76, 78.

${ }^{56}$ Labé, 1986 : 81.

${ }^{57}$ Fabri, 1969 : 64
} 
auditoire qui est loin d'être acquis à Folie qu'il défend, tout en réfutant les thèses de son adversaire Apolon.

L'exemple de concession rectificative qui suit permet d'illustrer la prise en compte des opinions de cet adversaire :

Il ne lui ha fait mal : neanmoins il s'en est mis en son plein devoir (p.83)

Il s'agit d'un cas de diaphonie ${ }^{58}$, dans lequel Mercure reprend, pour la contester, une affirmation utilisée dans le récit de l'altercation entre Amour et Folie, fait par Apolon :

Il la veut faire aymer : elle evite le coup (p.66)

Le procédé relève de la réfutation, ou confutation qui, selon Pierre Fabri, peut se faire « en conservant [la] prinse [de l'adversaire], mais on nye sa conclusion ${ }^{59}$. Il s'agit ici du lieu de l'ordre $^{60}$, dans lequel Mercure condamne un fait en mentionnant l'intention qui a présidé à sa réalisation.

$* * * * * * * * * * * * *$

L'importance de la concession dans le texte de Louise Labé est à mettre en relation avec le genre du débat, du dialogue, dans lequel la parole comme l'opinion de l'autre sont omniprésentes. La concession est une réaction, de quelque ordre qu'elle soit, à ces propos, réels ou supposés, de l'interlocuteur, singulier ou pluriel.

\section{Véronique MONTAGNE \\ Université de Nice Sophia-Antipolis}

\section{Bibliographie}

CHEVALIER J-C. (1968), Histoire de la syntaxe, naissance de la notion de complément dans la grammaire française (1530-1750), Genève, Droz.

FABRI P. (1969), Le grand et vrai art de pleine rhétorique, Genève, Slatkine Reprints.

LABE L. (1986), Oeuvres complètes, Paris, GF-Flammarion.

LORIAN A. (1973), Tendances stylistiques dans la prose narrative française du XVIème siècle, Paris, Klincksieck.

MARCHELLO-NIZIA C., Dire le vrai, l'adverbe si en français médiéval, Paris, Droz.

MEIGRET L. (1970), Tretté de la grammere francoeze, Genève, Slatkine reprints.

MOIGNET G. (1988), Grammaire de l'ancien français, Paris, Klincksieck.

MONTAGNE V. (2003), «L'expression hypotaxique et paratactique de la concession dans l'Heptaméron (1559) de Marguerite de Navarre », Français moderne, ${ }^{\circ} 2$.

MOREL M-A. (1996), La concession en français, Paris, Ophrys.

PALSGRAVE J. (1852), L'éclaircissement de la langue française, Paris, Imprimerie nationale.

PERELMAN C. et OLBRECHTS-TYTECA (1988), Traité de l'argumentation, éd. de l'université de Bruxelles.

RAMUS (1996), Dialectique, Paris, Vrin.

RAMUS (2001), Grammaire, Paris, Champion.

ROULET E. et alii (1985), dans L'articulation du discours en français contemporain, Peter Lang.

SOUTET O. (1992), La concession dans la phrase complexe en français, des origines au XVIème siècle, II, Genève, Droz.

\footnotetext{
${ }^{58}$ Roulet et al., $1985: 71$.

${ }^{59}$ Fabri, 1969 : 109.

${ }^{60}$ Perelman et Olbrechts-Tyteca, 1988.
} 